\title{
Prevenção Cardiovascular em Pacientes com Diabetes: Revisão Baseada em Evidências
}

revisão

\author{
Mariana Vargas Furtado \\ CARISI ANNE POLANCZYK
}

Serviço de Cardiologia do Hospital de Clínicas de Porto

Alegre, Departamento de

Medicina Interna da

Universidade Federal do Rio

Grande do Sul, Programa de

Pós-graduação em Medicina: Cardiologia, da UFRGS, Porto Alegre, RS.

Recebido em 06/01/07

Aceito em 11/01/07
RESUMO

Diabetes mellitus é uma doença associada com elevada incidência de doença aterotrombótica, especialmente cardíaca. Diversos estudos demonstraram que é possível reduzir a carga de doença nesta população através de estratégias preventivas. Este artigo revisa as evidências sobre a estimativa de risco cardiovascular nessa população e ações de prevenção, levando em consideração as principais recomendações na área. Modificações no estilo de vida, como dieta pobre em carboidratos, redução de peso e prática regular de atividade física, devem ser instituídas. Todo paciente de alto risco ou com evidências de doença cardiovascular deve manter sua glicemia $<100 \mathrm{mg} / \mathrm{dl}$ e $\mathrm{A} 1 \mathrm{c}<7 \%$. É recomendado uso diário de AAS em doses baixas e estatinas para controle de lípides, tendo como alvo $\mathrm{LDL}<100 \mathrm{mg} / \mathrm{dl}, \mathrm{HDL}>50 \mathrm{mg} / \mathrm{dl}$ e triglicerídios $<150 \mathrm{mg} / \mathrm{dl}$. O controle da pressão arterial deve ser buscado com medidas não-farmacológicas e antihipertensivos, sendo inibidores do sistema renina-angiotensina indicados na maioria dos casos, especialmente naqueles pacientes com nefropatia, associado a diuréticos tiazídicos na sua maioria para alcançar níveis tensionais-alvo < 130/80 mmHg. (Arq Bras Endocrinol Metab 2007;51/2:312-318)

Descritores: Diabetes mellitus; Doença cardiovascular; Prevenção primária

\section{ABSTRACT}

\section{Cardiovascular Prevention in Diabetic Patients: an Evidenced- based Review.}

Diabetes mellitus is a condition associated with cardiac complications, especially atherotrombotic disease. Several studies have demonstrated the importance of reducing cardiovascular burden on this population by adopting prevention strategies. This article revised clinical evidences on cardiovascular risk assessment and prevention actions, taking into consideration major recommendations in the field. Life-style changes with lowcarbohydrate diet, weight control, and regular physical activity must be implemented. High-risk patients or with established cardiovascular disease ought to have glycemic levels $<100 \mathrm{mg} / \mathrm{dL}$ and $\mathrm{A} 1 \mathrm{c}<7 \%$. It is recommended low dose of aspirin and statin for lipid management, targeting $\mathrm{LDL}<100 \mathrm{mg} / \mathrm{dL}, \mathrm{HDL}>50 \mathrm{mg} / \mathrm{dL}$ and tryglicerides $<150 \mathrm{mg} / \mathrm{dL}$. Blood pressure control with non-pharmacological and antihypertensive drugs must be instituted, favoring ACE inhibitors as first option, mainly in patients with renal disease, and combined with tiazides for the majority to achieve target blood pressure of $<130 / 80 \mathrm{mmHg}$. (Arq Bras Endocrinol Metab 2007;51/2:312-318)

Keywords: Diabetes mellitus; Cardiovascular disease; Primary prevention 
D IABETES MELLITUS (DM) É uma condição crítica, com elevado impacto individual e coletivo em termos de carga de doença cardiovascular. No Brasil, dados do Plano de Reorganização da Atenção à Hipertensão Arterial e ao DM de 2001 apontaram para uma prevalência na população brasileira acima de 40 anos de idade de 10\%, onde se estimou mais de 3,6 milhões de usuários do sistema público; quase metade desconhecia este diagnóstico e apenas $2 / 3$ destes indivíduos estão em acompanhamento nas unidades de atenção básica (1). Do ponto de vista clínico, é conhecido que a presença de DM confere um aumento no risco de desenvolver eventos circulatórios, sendo que até $80 \%$ dos indivíduos com DM vão desenvolver ou até mesmo morrer de doença macrovascular. As doenças do aparelho circulatório compreendem um espectro amplo de síndromes clínicas, mas têm nas doenças relacionadas à aterosclerose a sua principal contribuição, manifesta por doença arterial coronariana, doença cerebrovascular e de vasos periféricos, incluindo patologias da aorta, dos rins e de membros, com expressiva morbidade e impacto na qualidade de vida e produtividade dessa população. Inúmeros estudos epidemiológicos demonstraram, nas ultimas décadas, que a presença de alguns fatores de risco (hipertensão arterial sistêmica, diabetes, tabagismo, dislipidemia, história familiar, sedentarismo, obesidade central, ingesta pobre de frutas e vegetais e estresse psicossocial) explica quase $90 \%$ do risco atribuível de doença na população ao redor do mundo (2).

Em indivíduos adultos, o diabetes está associado a um alto risco de doença cardiovascular, em torno de 2 a 4 vezes maior em relação a indivíduos sem diabetes, sendo a doença cardiovascular (DCV) a principal causa de mortalidade e morbidade em indivíduos com diabetes tipo 2 (3). Diagnosticar e particularmente instituir um manejo agressivo dos fatores de risco em pacientes diabéticos é um dos aspectos mais centrais dos cuidados dessa população (4).

Há consenso sobre a importância da adoção de estratégias integrais, cada vez mais precoces ao longo do ciclo de vida, focadas na prevenção do DM e suas complicações. Nos últimos anos, evidências de inúmeros ensaios clínicos apontam para a necessidade da implementação de ações de saúde voltadas para um efetivo controle desses fatores de risco, visando à prevenção da doença e de seus agravos (3). Este texto aborda as evidências científicas disponíveis sobre estratégias de prevenção cardiovascular no indivíduo com DM, levando em consideração aqueles sem doença estabelecida ou prevenção primária. Essas estratégias permanecem após a manifestação de doença aterosclerótica como medidas de prevenção secundária, embora outras terapias possam ser adotadas dependendo da apresentação clínica.

\section{AVALIAÇÃO DE RISCO CARDIOVASCULAR}

Nos últimos anos, a prevenção cardiovascular tem sido baseada no conceito de risco cardiovascular global, que significa que os esforços para a prevenção de novos eventos cardiovasculares são orientados, não de maneira independente pelos riscos estabelecido pela elevação de fatores isolados como hipertensão arterial ou dislipidemia, mas pelo resultado da soma dos riscos imposta pela presença de múltiplos fatores, estimado pelo risco absoluto global de cada indivíduo (5).

Sob o enfoque preventivo, quanto maior o risco, maior o potencial benefício de uma intervenção terapêutica ou preventiva. O benefício de uma terapia na prevenção de desfechos não desejáveis pode ser expresso em termos relativos ou em termos absolutos, que levam em conta o risco individual ou a probabilidade de um indivíduo ter eventos em um período de tempo (6).

Indivíduos com DM, mesmo sem doença aterosclerótica estabelecida, têm um risco elevado de desenvolver eventos vasculares no futuro. Estima-se em torno de $20 \%$ a taxa de desenvolvimento de evento cardíaco em um período de 7 anos em pacientes diabéticos sem doença cardiovascular prévia (7). Por este motivo, sociedades internacionais (American Heart Association, American Diabetes Association), corroboradas pelas respectivas sociedades brasileiras, definem essa condição como sendo de "alto risco" para eventos cardiovasculares ou equivalente de "DCV estabelecida" para orientar medidas preventivas (8$10)$. Segundo escores de risco, isto equivale a uma estimativa superior a $20 \%$ em 10 anos de evento, ou 2-3\% ao ano. Entretanto, é conhecido que o risco absoluto para eventos macrovasculares não é uniforme em indivíduos com DM, e uma avaliação individualizada é necessária. Conforme o NHANES III (Third National Health and Nutrition Survey), pacientes diabéticos com síndrome metabólica e com mais de 50 anos possuem a maior prevalência de doença arterial coronariana $(19,2 \%)$ quando comparados a indivíduos que não apresentam ambos os diagnósticos. Entretanto, no grupo de pacientes com diabetes mas sem o diagnóstico de síndrome metabólica, a prevalência de DAC é similar à da população geral $(7,5 \%$ e $8,7 \%$ respectivamente). Como regra, indivíduos adultos jovens (< 40 anos) e crianças têm um risco baixo, nos primeiros 10 anos de doença, de apresentar esse tipo de 
complicação. Nestes casos, podem ser empregados algoritmos específicos para DM, validados em estudos clínicos, como escore de Framingham, UKPDS ou Diabetes PHD $(11,12)$.

\section{Recomendações}

A maioria dos adultos com diabetes tipo 1 e tipo 2 deve ser considerada com alto risco cardiovascular (grau A). A exceção compreende pacientes jovens, com curta duração da doença que não apresentem complicações cardiovasculares e sem outros fatores de risco cardiovasculares (grau A).

\section{ALIMENTAÇÃO SAUDÁVEL}

Mudanças de estilo de vida, como terapia de orientação nutricional e atividade física aeróbica, modificam o perfil lipídico, reduzem os níveis pressóricos e são centrais no manejo da glicemia e do controle de peso. Embora muito da abordagem dietética sempre tenha sido voltado ao controle do peso, ênfase maior tem sido dada para um controle adequado da glicemia e de outros fatores de risco (13).

A perda de peso em indivíduos obesos reduz a incidência da maioria dos fatores de risco para doença cardiovascular e melhora o controle da glicemia, sendo recomendada perda moderada $(7-10 \%$ peso corporal em 6 meses a 1 ano) em médio prazo. Até o presente não existem evidências de ensaios clínicos randomizados de que a perda de peso em indivíduos obesos tem efeito em redução de eventos cardiovasculares, mas estudo multicêntrico com poder adequado está em andamento (Look AHEAD).

Embora vários estudos buscassem identificar um macronutriente relacionado com proteção cardiovascular, até o momento isto não foi identificado. Porém, algumas intervenções nutricionais mostraram-se efetivas na redução de eventos cardiovasculares em indivíduos de alto risco em geral. Dietas protetoras são baseadas em alimentos de origem vegetal em abundância (frutas, hortaliças, pão, massa, cereais integrais, grãos e leguminosas), óleo de oliva e óleos vegetais (milho, soja, canola) como a principal fonte de gordura (com substituição de manteiga e cremes), carne vermelha em pouca quantidade e vinho em quantidades não mais do que moderadas (14). Salienta-se que as dietas são ricas em fibras e pobres em alimentos com carboidratos simples refinados e em comidas processadas comercialmente, com baixo teor de sal (DASH diet) (15). Além disso, um elemento importante nessas dietas é o teor mais elevado de ácidos graxos ômega 3, cuja ingestão aumentada está relacionada à redução de risco cardiovascular.

A suplementação alimentar com vitaminas antioxidantes, beta-carotenos, acido fólico ou vitamina B, ou ácidos graxos ômega-3 ou linoléico não se mostrou efetiva na prevenção primária cardiovascular e não é recomendada com base nas evidências disponíveis (13).

\section{Recomendações}

A perda de peso é recomendada para todos os pacientes com sobrepeso ou com obesidade que apresentem DM2 ou que estejam sob um maior risco de desenvolver a doença (grau B).

A abordagem primária para se atingir a perda de peso é a alteração do estilo de vida, a qual inclui não apenas a redução da ingestão calórica, mas aumento na atividade física (grau A).

Perdas modestas de peso da ordem de 5 a $10 \%$ trazem benefícios metabólicos significativos para o portador de DM (grau A).

\section{ATIVIDADE FÍSICA}

Exercício físico regular melhora o controle glicêmico, reduz fatores de risco cardiovasculares, contribui para perda de peso e pode prevenir diabetes em indivíduos de alto risco. Para alcançar estes objetivos, é recomendada a prática de, pelo menos, 150 minutos de exercício aeróbico de intensidade moderada (50-70\% da freqüência cardíaca máxima), distribuídos ao longo da semana, ou pelo menos 90 minutos de exercício intenso por semana. Na ausência de contra-indicações, deve ser encorajada a prática de exercício de resistência 3 vezes por semana, nos principais grupos musculares $(4,8)$.

\section{CONTROLE GLICÊMICO}

Evidências provenientes de ensaios clínicos randomizados demonstram de modo inequívoco que o controle glicêmico rígido reduz as complicações microvasculares da DM em longo prazo. Quando comparado a tratamentos convencionais, o controle rígido de glicemia baseado em diminuição das taxas de hemoglobina glicada (Alc) está associado à redução de complicações microvasculares em pacientes com diabetes tipo 1 e tipo 2. Níveis de Alc $>7 \%$ estão associados a um aumento de risco de complicações cardiovasculares, independentemente do tipo de tratamento utilizado. Entretanto, muita discussão existe sobre o real efeito do controle intensivo da glicemia nos desfechos cardiovasculares $(4,8,13)$. 
No estudo UKPDS, a relação entre risco cardiovascular e níveis de Alc se mostrou linear, para cada redução absoluta de $1 \%$ nos níveis da Alc há um declínio de $37 \%$ no risco das complicações microvasculares, mas o a redução de eventos cardiovasculares foi limítrofe (16). No acompanhamento de 6 anos do DCCD, tratamento intensivo com insulina reduziu eventos cardiovasculares. Outros estudos estão em andamento para testar esta hipótese. Apesar da falta dessas evidências, é recomendado um controle próximo ao normal dos níveis de glicemia pelos outros efeitos positivos comprovados.

Alguns estudos demonstram que a glicemia pós-prandial é mais efetiva em predizer risco de mortalidade em relação à glicemia de jejum. No estudo Diabetes Intervention, uma glicemia 1 hora pós-prandial $\leq 8,0 \mathrm{mmol} / \mathrm{dl}$ conferiu menor risco de infarto agudo do miocárdio e morte em pacientes com diabete tipo 2 , enquanto níveis $>10,0 \mathrm{mmol} / \mathrm{dl}$ estiveram associados com maior risco (17). Intervenções baseadas nesses dados precisam ainda ser comprovadas em desfechos clínicos relevantes.

\section{CONTROLE DA PRESSÃO ARTERIAL}

Hipertensão é uma condição comum em pacientes diabéticos, sendo o maior fator de risco para complicações cardiovasculares e microvasculares, como cardiopatia isquêmica, nefropatia e retinopatia. No diabetes tipo 1 , a hipertensão geralmente resulta do desenvolvimento de nefropatia, enquanto que no diabetes tipo 2 a hipertensão mais freqüentemente está presente como parte da síndrome metabólica.

Os níveis de pressão arterial (PA) considerados alvos a serem atingidos estão baseados em ensaios clínicos que demonstraram benefícios em redução da pressão sistólica $<130 \mathrm{mmHg}$ e $<80 \mathrm{mmHg}$ em indivíduos com diabetes. Estudos como o UKPDS 38 e Hypertension Optimal Treatment (HOT) demonstraram, de maneira consistente, que a pressão diastólica de $80 \mathrm{mmHg}$ deve ser almejada, apresentando redução clínica importante nas complicações macrovasculares, mortalidade cardiovascular e morte relacionada ao diabetes (grau B) (18). As evidências para a meta da pressão sistólica $<130 \mathrm{mmHg}$ são mais fracas e estão baseadas em 2 estudos de coorte e no ensaio clínico Normotensive Appropriate Blood Pressure Control in Diabetes (ABCD), no qual observou-se uma relação direta entre níveis altos de pressão sistólica e mortalidade, doença arterial coronariana e nefropatia (grau C) $(19,20)$. Baseados nestas evidências, as diretrizes internacionais mais recentes recomendam como meta a ser atingida uma $\mathrm{PA}<130 / 80 \mathrm{mmHg}$.
Pacientes diabéticos que não apresentam sinais de nefropatia podem ser inicialmente tratados com modificação no estilo de vida (dieta e atividade física) por um período de 3 meses. Se a PA não for controlada após esse período e naqueles pacientes com diabetes e sinais de nefropatia, o tratamento com drogas anti-hipertensivas deve ser iniciado. As drogas recomendadas para início do tratamento são inibidores da enzima conversora da angiotensina (ECA), bloqueadores do receptor de angiotensina, $\beta$-bloqueadores e diuréticos tiazídicos (13). Esta recomendação resulta de ensaios clínicos randomizados que compararam agentes dessas classes com placebo ou com outra droga ativa, demonstrando redução de mortalidade e morbidade cardiovascular em pacientes diabéticos. Os bloqueadores de canal de cálcio não foram estudados em ensaios com esse tipo de delineamento, mas demonstraram eficácia em análises posthoc de um ensaio clínico, por isso são considerados opção em pacientes que não toleram as 4 classes inicialmente citadas (21).

$\mathrm{O}$ estudo Antibypertensive and Lipid-Lowering Treatment to Prevent Heart Attack Trial (ALLHAT), um grande ensaio clínico contendo > 12.000 pacientes com diabetes randomizados para tratamento com inibidor do cálcio, inibidor da ECA, diurético tiazídico e bloqueador $\alpha$-adrenérgico, não encontrou diferenças em desfechos cardiovasculares em 5 anos de seguimento entre os fármacos anlodipina, lisinopril e clortalidona (22). O diurético foi moderadamente mais efetivo do que os outros agentes, particularmente em reduzir as taxas de insuficiência cardíaca. $\mathrm{O}$ grupo de tratamento do bloqueador $\alpha$-adrenérgico, doxazosin, foi suspenso precocemente por apresentar um excesso de casos de insuficiência cardíaca em relação ao grupo do diurético. Por esta razão, os bloqueadores $\alpha$ adrenérgicos são evitados como primeira linha de tratamento anti-hipertensivo em pacientes diabéticos.

Diversos estudos sugerem uma superioridade dos inibidores da ECA em relação aos antagonistas do cálcio em reduzir eventos cardiovasculares. Alguns estudos de menor poder e meta-análises sugerem um benefício com inibidores da ECA ou bloqueadores dos receptores (ARA-2), não completamente atribuído à redução da pressão arterial, na prevenção da doença renal, sendo considerados classes de primeira escolha nesta população $(23,24)$. Por outro lado, é conhecido que a maioria dos indivíduos necessita mais de uma classe anti-hipertensiva para controle adequado dos níveis tensionais, e os diuréticos tiazídicos em baixas doses são uma das primeiras drogas a serem consideradas (25). 


\section{Recomendações}

Pacientes diabéticos sem nefropatia com PA > 130/80 $\mathrm{mmHg}$ devem receber drogas anti-hipertensivas adicionadas à modificação do estilo de vida. As seguintes drogas são recomendadas como primeira linha:

- Inibidor da ECA (grau A);

- Bloqueadores do receptor da angiotensina (grau A para pacientes com hipertrofia de ventrículo esquerdo, HVE; grau B se HVE não estiver presente);

-Diurético tiazídico (grau A);

- $\beta$-bloqueador (grau B);

- Bloqueador do canal de cálcio (grau B);

Em pacientes diabéticos tipo l com HAS e com qualquer grau de albuminúria, inibidores da ECA retardam a progressão para nefropatia (grau A);

Em pacientes diabéticos tipo 2 com HAS e microalbuminúria, inibidores da ECA e bloqueadores do receptor da angiotensina retardam a progressão para macroalbuminúria (grau A).

\section{CONTROLE DA DISLIPIDEMIA}

O distúrbio de lípides mais comum em diabetes tipo 2 é a hipertrigliceridemia (TG), baixa taxas de colesterol HDL e concentrações normais de LDL. Entretanto, com a hiperglicemia crônica há uma maior oxidação e glicação das partículas de LDL, com acúmulo de partículas densas de LDL aumentando sua aterogenicidade.

Em uma coorte canadense que acompanhou 2.473 pacientes com diabetes, encontrou-se uma prevalência de dislipidemia de $55 \%$ em pacientes com 2 anos de doença e esta proporção aumentou para $66 \%$ após 15 anos (26).

O manejo da dislipidemia com redução do LDL colesterol e triglicerídios e aumento do HDL tem se mostrado eficaz na redução de doença macrovascular e mortalidade em pacientes com diabetes tipo 2 , principalmente naqueles pacientes com eventos cardiovasculares prévios.

O estudo Heart Protection Study (HPS) demonstrou que o uso diário de $40 \mathrm{mg}$ de sinvastatina resultou em uma redução de $27 \%$ na taxa de eventos cardiovasculares e de $25 \%$ na taxa de acidentes vasculares cerebrais quando comparados com placebo. $\mathrm{O}$ benefício do tratamento foi independente dos níveis iniciais de HDL e LDL e da presença ou não de diabetes (27).

O estudo Collaborative Atorvastatin Diabetes Study (CARDS) foi o primeiro ensaio clínico realizado exclusivamente em pacientes diabéticos sem doença cardiovascular estabelecida. O tratamento com atorvastatina $10 \mathrm{mg}$ esteve associado com uma redução de 37\% nos eventos cardiovasculares e $48 \%$ nos acidentes vasculares cerebrais (AVC) em comparação com placebo (28).

Recentemente, uma meta-análise com $>90.000$ pacientes tratados com estatinas demonstrou que, para cada redução de $1,0 \mathrm{mmol} / \mathrm{L}(38 \mathrm{mg} / \mathrm{dl})$ nos níveis de LDL, há uma redução em $20 \%$ na taxa de eventos cardiovasculares, independentemente dos valores basais de LDL (29). Esta relação linear entre risco cardiovascular e redução de níveis de LDL sugere não haver alvo de tratamento nem limite para redução de LDL. Entretanto, os autores sugerem valor-alvo de LDL de $70 \mathrm{mg} / \mathrm{dl}$.

Uma terapia combinada de estatina e fibrato ou estatina e niacina parece ser eficaz em pacientes que necessitam tratamento para as 3 frações de lípides, principalmente redução dos triglicerídios. Embora o uso dessas medicações pareça racional no controle laboratorial, os dados de ensaios clínicos não são consistentes em relação ao benefício do controle dos triglicerideos com fibratos ou do HDL com niacina. Além disso, associações com estatinas não foram testadas e aumentam o risco de elevação dos níveis de transaminases, miosites e rabdomiólises. Essa forma de dislipidemia parece ser mais suscetível a modificações do estilo de vida (atividade física e dieta) e ao melhor controle glicêmico. Se, após 4 a 6 meses de instituição de dieta e atividade física e uso diário de estatina, os níveis de HDL permanecerem < $40 \mathrm{mg} / \mathrm{dl}$, alguns autores recomendam o tratamento com niacina $(8,13)$. No estudo HATS (HDL Atherosclerosis Treatment Study), a combinação de baixas doses de sinvastatina $(10$ a $20 \mathrm{mg} /$ dia $)$ com altas doses de niacina ( 2 a $4 \mathrm{~g} /$ dia) esteve associada com redução absoluta de risco de $13 \%$ para desfechos cardiovasculares (30). Com relação à hipertrigliceridemia, há poucas evidências que suportem metas a serem atingidas. O uso de fibratos está recomendado principalmente para reduzir o risco de pancreatite em pacientes com triglicerídios $>400-500 \mathrm{mg} / \mathrm{dl}$ que não respondem ao controle glicêmico, perda de peso e restrição de carboidratos. Apesar de estudos mostrarem prevenção de eventos cardiovasculares com monoterapia de fibratos, não há evidências que suportem o uso combinado de estatina e fibratos na redução de risco cardiovascular em pacientes com diabetes.

\section{Recomendações}

Adultos com alto risco cardiovascular devem ser tratados com estatina, almejando níveis de LDL $<100$ $\mathrm{mg} / \mathrm{dl}$ (Grau A).

Em adultos, o primeiro alvo da terapia são os níveis de LDL (grau $\mathrm{A}$ ), secundariamente às taxas de HDL e triglicerídios (grau D, consensos). 
Para pacientes que não atingem níveis ótimos apesar da terapia com estatinas, a associação de drogas deve ser considerada:

-Estatina + ezetimibe (grau B);

-Estatina + fibrato (grau B);

- Estatina + niacina (grau B);

\section{TERAPIA ANTIPLAQUETÁRIA}

Pacientes com diabetes possuem uma variedade de alterações na função plaquetária que predispõem à agregação plaquetária e à síntese de tromboxane. $\mathrm{O}$ uso de aspirina (AAS) tem sido recomendado como prevenção primária e secundária de eventos cardiovasculares em pacientes diabéticos. Uma meta-análise envolvendo número expressivo de ensaios clínicos demonstrou a eficácia da aspirina em reduzir eventos cardiovasculares com taxas de redução e $30 \%$ de IAM e $20 \%$ de AVC. A AAS parece ser mais efetiva do que outros agentes antiplaquetários (31). Não há evidências que suportem nenhuma dose específica, a maioria dos estudos utiliza doses entre 80 e 325 $\mathrm{mg} /$ dia, sendo consenso utilizar a menor dose possível para limitar os efeitos adversos (32). Pacientes que não tolerarem o uso de AAS devem substituir por outro agente antiplaquetário como clopidogrel. Os agentes antiplaquetários não devem ser utilizados em pacientes com história recente de sangramento gástrico ou insuficiência renal e hepática importantes. AAS está contra-indicada em pacientes < 21 anos pelo risco aumentado de desenvolver Síndrome de Reye (13).

\section{Recomendação}

Baixas doses de AAS (80 a $325 \mathrm{mg} /$ dia) estão indicadas a todos os pacientes com diabetes e evidências de doença cardiovascular, e naqueles sem doença estabelecida mas com outros fatores de risco (história familiar de DAC, hipertensão, tabagismo, dislipidemia e albuminúria) (grau $\mathrm{A}$ ).

\section{SUMÁRIO DAS RECOMENDAÇÕES}

Pacientes diabéticos devem ter estimado seu risco cardiovascular. Modificações no estilo de vida, como dieta pobre em carboidratos, redução de peso e prática regular de atividade física, devem ser instituídas. Todo paciente de alto risco ou com evidências de doença cardiovascular deve manter sua glicemia $<100 \mathrm{mg} / \mathrm{dl}$ e Alc $<7 \%$. É recomendado uso diário de AAS em doses baixas e estatinas para controle de lípides, tendo como alvo $\mathrm{LDL}<100 \mathrm{mg} / \mathrm{dl}$, HDL $>50 \mathrm{mg} / \mathrm{dl} \mathrm{e}$ triglicerídios $<150 \mathrm{mg} / \mathrm{dl}$. Deve-se buscar uma pressão arterial $<130 / 80 \mathrm{mmHg}$ com uso de medidas nãofarmacológicas e anti-hipertensivos, sendo inibidores do sistema renina-angiotensina indicados naqueles pacientes com sinais de nefropatia.

\section{REFERÊNCIAS}

1. Brasil. Ministério da Saúde. Secretaria de Políticas de Saúde. Departamento de Ações Programáticas Estratégicas. Plano de reorganização da atenção à hipertensão arterial e ao diabete melito: hipertensão arterial e diabete melito. Departamento de Ações Programáticas Estratégicas. Brasília: Ministério da Saúde, 2001. p. 104.

2. Yusuf S, Hawken S, Ounpuu S, Dans T, Avezum A, Lanas F, et al.; INTERHEART Study Investigators. Effect of potentially modifiable risk factors associated with myocardial infarction in 52 countries (the INTERHEART study): case-control study. Lancet 2004;364:937-52.

3. Brasil. Ministério da Saúde. Secretaria de Políticas de Saúde. Departamento de Atenção Básica. Diabetes Mellitus/ Ministério da Saúde, Secretaria de Atenção à Saúde, Departamento de Atenção Básica. Brasília: Ministério da Saúde, 2006. p. 56.

4. IDF Clinical Guidelines Task Force. Global guideline for Type 2 diabetes. Brussels: International Diabetes Federation, 2005.

5. Duncan BB, Schmidt MI, Giugliani ERJ. Medicina ambulatorial: condutas de atenção primária baseadas em evidências. 3a ed. Porto Alegre: Artmed, 2004. p. 1577.

6. Brasil. Ministério da Saúde. Secretaria de Políticas de Saúde. Departamento de Atenção Básica. Prevenção clínica de doenças cardiovasculares e renais. Ministério da Saúde, Secretaria de Atenção à Saúde, Departamento de Atenção Básica. Brasília: Ministério da Saúde, 2006. p. 56.

7. Canadian Diabetes Association. Clinical Practice Guidelines Expert Commitee. Dyslipidemia in Adults with Diabetes. Can J Diabetes 2006;30(3):230-40.

8. Executive Summary of The Third Report of The National Cholesterol Education Program (NCEP) Expert Panel on Detection, Evaluation, And Treatment of High Blood Cholesterol In Adults (Adult Treatment Panel III). JAMA 2001;285:2486-97.

9. Standards of Medical Care in Diabetes (Position Statement) American Diabetes Association. Diabetes Care 2005;28(suppl 1):S4-36.

10. Consenso Brasileiro Sobre Diabetes - 2002. Diagnóstico e Classificação do Diabetes Melito e Tratamento do Diabetes Melito do Tipo 2. Sociedade Brasileira de Diabetes (SBD), 2002.

11. Risk assessment tool for estimating your 10-year risk of having a heart attack. National Heart, Lung and Blood Institute. Disponível em: <http://hin.nhlbi.nih.gov/atpiii/calculator.asp?usertype=pub>. Acessado em: 26 julho 2003.

12. Estimating Coronary Heart Disease (CHD) Risk Using Framingham Heart Study Prediction Score Sheets. National Heart, Lung and Blood Institute. Disponível em: <http://www.nhlbi.nih.gov/about/framingham/riskabs.htm>. Acessado em: 12 setembro 2003.

13. Buse JB, Ginsberg HN, Bakris GL, Clark NG, Costa F, Eckel R, et al.; American Heart Association; American Diabetes Association. Primary Prevention of Cardiovascular disease in patients with diabetes. Diabetes Care 2007;30(1):162-72.

14. Singh RB, Dubnov G, Niaz MA, Ghosh S, Singh R, Rastogi SS, et al. Effect of an Indo-Mediterranean diet on progression of coronary artery disease in high risk patients (Indo-Mediterranean Diet Heart Study): a randomised single-blind trial. Lancet 2002;360:1455-61. 
15. Chobanian AV, Bakris GL, Black HR, Cushman WC, Green LA, Izzo JL, Jr. et al. The Seventh Report of the Joint National Committee on Prevention, Detection, Evaluation, and Treatment of High Blood Pressure: the JNC 7 report. JAMA 2003;289:2560-72.

16. Intensive Blood Glucose Control With Sulphonylureas or Insulin Compared With Conventional Treatment and Risk of Complications in Patients With Type 2 Diabetes. UK Prospective Diabetes Study Group (UKPDS). Lancet 1998;352:83753.

17. Hanefeld M, Fischer S, Julius $U$, Schulze J, Schwanebeck $U$, Schmechel H, et al. Risk factors for myocardial infarction and death in newly detected NIDDM: the Diabetes Intervention Study, 11-year follow-up. Diabetologia 1996;39:1577-83.

18. Hansson L, Zanchetti A, Carruthers SG, Dahlof B, Elmfeldt D, Julius $\mathrm{S}$, et al. Effects of intensive blood-pressure lowering and low-dose aspirin in patients with hypertension: principal results of the Hypertension Optimal Treatment (HOT) randomized trial. Lancet 1998;351:1755-62.

19. Orchard TJ, Forrest KY, Kuller LH, Becker DJ; Pittsburgh Epidemiology of Diabetes Complications Study. Lipid and blood pressure treatment goal for type 1 diabetes: 10-year incidence data from the Pittsburg Epidemiology of Diabetes Complications Study. Diabetes Care 2001;24:1053-9.

20. Adler AI, Stratton IM, Neil HA, Yudkin JS, Matthews DR, Cull CA, et al. Association of systolic blood pressure with macrovascular and microvascular complication of type 2 diabetes (UKPDS 36): prospective observational study. BMJ 2000;321:412-9.

21. Tuomilehto J, Rastenyte D, Birkenhager WH, Thijs L, Antikainen $\mathrm{R}$, Bulpitt $\mathrm{CJ}$, et al. Effects of calcium-channel blockade in older patients with diabetes and systolic hypertension. N Engl J Med 1999;340:677-84.

22. The ALLHAT Officers and Coordinators for the ALLHAT Collaborative Research Group. Major cardiovascular events in hypertensive patients randomized to doxazosin vs chlorthalidone. The Antihypertensive and Lipid- Lowering Treatment to prevent Heart Attack Trial (ALLHAT). JAMA 2000; 283:1967-75.

23. Yusuf S, Sleight $P$, Pogue J, Bosch J, Davies R, Dagenais G. Effects of an angiotensin-converting-enzyme inhibitor, ramipril, on cardiovascular events in high-risk patients. The Heart Outcomes Prevention Evaluation Study Investigators. N Engl J Med 2000;342:145-53.

24. Casas JP, Chua W, Loukogeorgakis S, Vallance P, Smeeth L, Hingorani $A D$, et al. Effect of inhibitors of the reninangiotensin system and other antihypertensive drugs on renal outcomes: systematic review and meta-analysis. Lancet 2005;366:2026-33.
25. Fuchs FD. Hipertensão arterial sistêmica. In: Duncan BB, Schmidt MI, Giugliani E (eds). Medicina Ambulatorial: condutas em atenção primária baseadas em evidências. 3a ed. Porto Alegre: Artmed, 2004. pp. 641-56.

26. Harris SB, Ekoé J-M, Zdanowicz Y, Webster-Bogaert S. Glycemic control and morbidity in the Canadian primary care setting (results of the diabetes in Canada evaluation study). Diabetes Res Clin Pract 2005;70:90-7.

27. MRC/BHF Heart Protection Study of cholesterol lowering with sinvastatin in 20,536 high-risk individuals: a randomised placebo-controlled trial. Lancet 2002;360:7-22.

28. Colhoun HM, Betteridge DJ, Durrington PN, Hitman GA, Neil HA, Livingstone SJ, et al.; CARDS investigators. Primary prevention of cardiovascular disease with atorvastatin in type 2 diabetes in the Collaborative Atorvastatin Diabetes Study (CARDS): Multicentre randomised placebo-controlled trial. Lancet 2004;364:685-96.

29. Baigent C, Keech A, Kearney PM, Blackwell L, Buck G, Pollicino C, et al.; Cholesterol Treatment Trialists' (CTT) Collaborators. Efficacy and safety of cholesterol-lowering treatment: prospective meta-analysis of data from 90,056 participants in 14 randomised trials of statins. Lancet 2005;366:1267-78.

30. Brown BG, Zhao XQ, Chait A, Fisher LD, Cheung MC, Morse JS, et al. Sinvastatin and niacin, antioxidant vitamins, or the combination for the prevention of coronary disease. N Engl J Med 2001;345:1583-92.

31. Collaborative meta-analysis of randomised trials of antiplatelet therapy for prevention of death, myocardial infarction, and stroke in high-risk patients. BMJ 2002;324:71-86.

32. Hayden M, Pignone M, Phillips C, Mulrow C. Aspirin for the primary prevention of cardiovascular events: a summary of the evidence for the U.S. Preventive Services Task Force. Ann Intern Med 2002;136:161-72.

\section{Endereço para correspondência:}

Carisi A. Polanczyk

Hospital de Clínicas de Porto Alegre

Rua Ramiro Barcelos 2350, sala 2060

90035-903 Porto Alegre, RS

E-mail: cpolanczyk@hcpa.ufrgs.br 